\title{
AGUSTIN MADAN, PROFESOR, CANÓNIGO Y ASESOR DE CAMPOMANES
}

\author{
POR \\ Teresa GonZÁlez Pérez \\ Catedrática. Facultad de Educación. Universidad de La Laguna
}

\section{RESUMEN}

Pretendemos indagar en la vida del erudito Agustín Madan (1739-1796), un eclesiástico canario avanzado en el tiempo y destacado en los círculos culturales madrileños, pero también influyente en la vida del historiador, científico y educador José de Viera y Clavijo. Sus ansias de saber le enfrentaron con el Tribunal de la Santa Inquisición y con el Cabildo Catedral. Acercarse al erasmismo a través de lecturas prohibidas y pretender profesionalizarse le trajeron muchos problemas, pero no renunció a sus propósitos y obtuvo la primera cátedra de Hebreo de los Reales Estudios de San Isidro de Madrid. Por sus amplios conocimientos fue requerido por el ministro Pedro de Campomanes para que le asesorara sobre los estudios lingüísticos y al le propuso algunas soluciones para promocionar dichos estudios. Madan incomprendido por sus contemporáneos no recibió ni ha recibido el reconocimiento que merece.

PALABRAS ClAVE: Siglo dieciocho, Agustín Madan, ilustrado, profesor, lenguas, eclesiástico, inquisición, Cabildo Catedral, asesor, Campomanes

\section{AGUSTÍN MADAN, PROFESSOR, CANON AND ADVISER TO CAMPOMANES}

\begin{abstract}
In this article we study the life of the learned Agustín Madan (1739-1796), a churchman from the Canary Islands who was very ahead of his times and noted in Madrid cultural circles. He was also very influential in the life of José de Viera y Clavijo, a historian, scientist and educator. His desire for learning put him in
\end{abstract}


conflict with the Tribunal of the Spanish Inquisition and with the Chapter of the Cathedral. His leanings derived from the followers of Erasmus developed after reading forbidden books and his desire to become more professional brought him many problems. However, he never went back on his plans and eventually became the first holder of the Hebrew Chair of Royal Studies of Saint Isidor of Madrid. Because of his broad knowledge, he was made adviser on linguistic studies by Pedro de Campomanes. Madan suggested to him several ideas which helped popularize linguistic studies. Madan was misunderstood by his contemporaries and did not and has not received the recognition that he deserves.

KEY WORDS: Eighteen century, Agustín Madan, learned, professor, languages, churchman, inquisition, Chapter of the Cathedral, adviser, Campomanes

Recibido/Received 11-03-2009

Aceptado/Accepted 07-13-2010

\section{INTRODUCCIÓN}

La vida del eclesiástico Agustín Madan transcurrió en la segunda mitad del «siglo de las luces» y su interés por la cultura y mejorar el saber sintonizaba perfectamente con la idea de los ilustrados de disipar las tinieblas de la vida humana. A pesar de ello su trayectoria vital no fue fácil. Vivió un ambiente lleno de dificultades debido a su amplitud de miras que iban más allá del sacerdocio y su compromiso eclesiástico. El interés por la cultura quedó patente en su propia biblioteca repleta de libros, muchos de ellos prohibidos, que entroncaban con planteamientos renovadores y tropezaban con el comportamiento conservador de la Iglesia en Canarias. El espíritu de liberalización ante la escolástica y la apertura a las corrientes modernas de pensamiento aportaron un sello indiscutible a su cualificada preparación intelectual.

El interés contrastado de este ilustrado por las publicaciones extranjeras de la época nos sorprende. Ni la insularidad ni el aislamiento español, constituían una barrera que impedía la penetración de la bibliografía extranjera en el archipiélago canario. Así, una minoría, entre ellos el erudito bibliófilo Agustín Madan, seguía las publicaciones culturales y religiosas europeas con verdadero interés. El espíritu enciclopedista europeo se afianzó en España a través de la abundante bibliografía que traspasó las fronteras y burló los controles burocráticos de censura. Por ello, a pesar de la rígida actuación de los Tribunales de la Inquisición quemando libros y persiguiendo a sus lectores, este movimiento no dejó de tener su consonancia con Europa.

Analizando el grueso de volúmenes que conformaron su biblioteca personal podemos observar su profundo sentido religioso y el contacto con la espiritualidad europea. Su amplia información y repertorio bibliográfico conectaba con la 
mentalidad europea del XVIII. Con el influjo de estas corrientes de pensamiento Madan adoptó una postura que conectaba con los ilustrados católicos de Francia, Italia, Inglaterra, Alemania y por supuesto de España. Compartió sus ideas, leyó idénticos libros y siguió con curiosidad el proceso y evolución del pensamiento europeo. Testimonio de ello fueron las obras galicanas que engrosaban su biblioteca, entre ellas cabe citar a Bousset, Fleury y Alexandre. Su desmesurado interés por el movimiento jansenista le condujo a las lecturas de Van Espen. La tenencia de libros prohibidos atestigua su pluralidad intelectual, sufriendo persecución por tener un pensamiento diferenciado y otra formación cultural.

Tal formación chocaba con la mentalidad clerical imperante en Canarias y sus inquietudes culturales se vieron interferidas por un proceso inquisitorial. Históricamente la Iglesia ha sido reaccionaria incluso con sus propios miembros, sobre todo, cuando éstos desafiaban sus rígidos moldes. Al hilo de lo anterior, una mirada retrospectiva nos sitúa en la incomprensión e intransigencia del Cabildo Catedral de Canaria que le generaron dificultades hasta el extremo de ser amonestado y sancionado. Sin embargo no se arredraba ante las continuas censuras que recibía de la jerarquía eclesiástica, no se amilanaba ante el conservadurismo y la prepotencia clerical. Mantuvo una perpetua lucha entre sus intereses culturales y su puesto eclesiástico. Cabalgaba entre dos mundos culturales dispares: la iglesia canaria empapada por el conservadurismo y la cultura ilustrada que le sugestionaba. Perseguido por leer libros prohibidos no renegó de su fe cristiana. Era una persona de profundas convicciones religiosas que incluso las resalta a la hora de revalorizar la lengua hebrea. Tampoco dudó de su capacidad intelectual y de su valía personal para desempeñar cargos oficiales, como el caso de pretender una plaza en el Tribunal de la Santa Inquisición. Sin embargo, su petición se desestimó injustamente. No se le valoraba y se oponían a su promoción. Le marginaban ante su afán desdeñado por los libros y los conocimientos.

Agustín Madan fue un erudito canario que en Madrid alternaba con destacados intelectuales y frecuentaba las tertulias que reunían a expertos en las lenguas clásicas. Cualificado y preocupado por la renovación del conocimiento de las lenguas clásicas, fue el primer catedrático de Hebreo de los Reales Estudios de San Isidro de Madrid. Divulgador de los nuevos planteamientos de las lenguas clásicas, deseoso de que progresaran sus estudios, puso todo su empeño en la docencia del hebreo. En su pensamiento y actividad educativa destacaba su interés por desplazar la ignorancia lingüística. $\mathrm{Al}$ mismo tiempo su ideario educativo desveló claves de su pensamiento cristiano, pues en la práctica educativa incluyó como contenido fundamental el conocimiento de la Sagrada Biblia, forma de aprendizaje de la lengua hebrea. Su cualificación quedó probada y, no en vano, fue requerido por Pedro de Campomanes para que realizara un estudio sobre la forma de promocionar las lenguas clásicas. 
No obstante lo anterior y a pesar de su amplio campo de actividad -funciones eclesiásticas, docencia, representante del cabildo catedral en Madrid- fue tan incomprendido en su tiempo como ignorado en las generaciones sucesivas, igual que ha sucedido a otros tantos eruditos isleños. Somos conscientes de que la memoria histórica ha sido selectiva, pero cuando se trata de personas a veces resulta injusta y no siempre resalta su actividad en la dimensión que corresponde. De esta manera, olvida a unos, denigra a otros y enaltece a aquellos que tuvieron más eco. A veces las personas más nombradas no coinciden con las más valiosas, claro que no ocurre sólo en el pasado, también en el presente. En algunos casos tanto la historiografía como los investigadores han caído en los tópicos, sin embargo, decantarse por estos no contribuye a despejar incógnitas, silenciarlas y ocultarlas tampoco. Agustín Madan en la historiografía es poco conocido, más bien ha sido un desconocido. Ni durante el siglo XVIII ni en nuestros días, ha sido reconocido como merece, otros con menos méritos han resulado más valorados. Si bien se trata de una de las figuras representativas del siglo XVIII canario ha permanecido ignota a través del tiempo.

El objetivo de este trabajo es dar a conocer al profesor canario Agustín Madan, que fue primer catedrático de Hebreo de los Reales Estudios de San Isidro de Madrid, además de canónigo y diputado del Cabildo Catedral de Canaria, que por sus conocimientos fue requerido por el ministro Pedro de Campomanes para que le asesorara sobre los estudios lingüísticos. Pretendemos esbozar en este análisis de su personalidad y su labor como profesional de la docencia con el propósito de penetrar en su pensamiento en el intento de aportar una visión de conjunto de su persona, de sus conocimientos y la valoración de su actitud intelectual. En cuanto a la metodología hemos seguido el método científico de investigación histórica.

La documentación se halla dispersa entre distintos fondos documentales. Por tal motivo ha sido preciso rastrear en diversos archivos insulares y peninsulares. Este interés por conocer su pensamiento y su actividad ha tropezado con serios y profundos obstáculos ante la dificultad de abarcar su fecunda y múltiple actividad. Ha sido necesario seguir minuciosamente su correspondencia, observar la exposición de sus ideas críticas, leer las respuestas de sus interlocutores y seguir el proceso del influjo de su pensamiento a través de las actas capitulares, tanto en sus períodos de ausencia y estancia en Madrid como cuando reingresó en el cuerpo capitular (con el que mantiene notadas discrepancias). $\mathrm{Su}$ correspondencia nos esclareció su personalidad y verdadera importancia en el ambiente cultural del siglo XVIII. Sus cartas son una valiosa fuente de información sobre su formación, su ámbito sociofamiliar y sus preocupaciones, donde se vislumbran las situaciones que tuvo que afrontar. El carácter disperso de la documentación inédita, conservada en diferentes fondos, dificultó la investigación. Dificultades que no sólo se refieren al aporte documental sino también a la bibliografía específica.

Hispania Sacra, LXIII

127, enero-junio 2011, 185-204, ISSN: 0018-215-X 
Realizamos un rastreo en diferentes archivos nacionales y locales, donde localizamos diversos documentos como fuentes de información. Entre los diferentes depósitos documentales consultados citamos el Archivo de la Fundación Universitaria Española, el Archivo General de Simancas, el Archivo Histórico Nacional, el Archivo de la Catedral de Santa Ana de Las Palmas, el Archivo Histórico Diocesano de Las Palmas de Gran Canaria, el Archivo Parroquial de Nuestra Señora de la Peña de Francia (Puerto de la Cruz-Tenerife), el Archivo de la Real Sociedad Económica de Amigos del País de Tenerife y el Archivo de El Museo Canario (Las Palmas).

\section{APUNTE BIOGRÁFICO. EL INICIO DE SU FORMACIÓN Y EL DESARROLLO DE SU TALENTO}

Agustín Madan nació ${ }^{~}$ el 14 de abril de 1739, en el Puerto de la Orotava (actual Puerto de la Cruz -Tenerife) y falleció ${ }^{2}$ en Las Palmas de Gran Canaria el 27 de julio de 1796. Su familia residió en el citado Puerto de la Orotava hasta 1741, año en que se trasladó a La Laguna. En esta ciudad creció al tiempo que recibió una esmerada educación en el convento de los agustinos. Desde muy temprana edad inclinó su formación intelectual hacia la vida religiosa. ${ }^{3}$ En 1756 recibió las Ordenes Menores de manos del obispo de Canarias Fray Valentín Morán ${ }^{4}$ y en 1760 fue ordenado de subdiácono. ${ }^{5}$ En estas fechas se convirtió en caudatario y familiar del citado obispo. Más tarde, en 1761 el prelado tuvo que renunciar a la mitra por problemas de salud regresando a Asturias su tierra natal. Agustín Madan viajó con él y aprovechó la oportunidad para completar sus estudios, ante la imposibilidad de hacerlo en Canarias. De este modo estudió cinco años de estudios mayores en la universidad de Oviedo y se graduó en la misma Universidad de Bachiller de Leyes y Cánones durante los cursos 1762-63. En Canarias los aspirantes al ingreso en el estado eclesiástico tenían que instruirse en los conventos y aplicarse individualmente en los estudios, ya que en las islas no había seminarios ni otra forma de promocionar en sus estudios. ${ }^{6}$ El propio Cabildo informaba que el estudio era el único premio para las prebendas y que luego les servía para otros empleos en las islas, España y América.

\footnotetext{
1 A.P.N.S.P.F. Libro de Bautismos no 6 (1738-1754). Extracto de la Partida de Bautismo. Folio 13 vto. Año 1739.

2 A.C.S.A.L.P.G.C. Libro de defunciones, $\mathrm{n}^{\circ} 7$.

${ }^{3}$ A.H.D.L.P.G.C. Sección 11. Expediente de Ordenaciones. Legajo 35. Años 1751-1760.

${ }^{4}$ A.H.D.L.P.G.C. Ordenamientos 1751-1761. Folios 40 rto. 41 vto.

5 A.H.D.L.P.G.C. Ibidem, fol. 68 vto.

${ }^{6}$ A.C.S.A.L.P.G.C. Archivo Secreto. Legajo 25 - n ${ }^{\circ} 11$.
} 
Con posterioridad se trasladó a Madrid donde se instruyó en lenguas clásicas y aprendió la lengua hebrea. Hemos localizado un dato que prueba su presencia en Madrid, antes de obtener una ración en la Catedral de Canarias. En 1766, por orden de los Alcaldes de Casa y Corte, se realizó una lista de los curas que se hallaban en la corte y cuyo destino era otro. El control del clero fue otra de las medidas regalistas que emprendió la corona, de forma que los sacerdotes que no desempeñaran una misión concreta y comprobable debían reincorporarse a sus destinos. En esta relación de clérigos se incluyó a Agustín Madan que residía en la calle del Prado junto con Diego Eduardo. ${ }^{7}$ Ante su requerimiento explicó que se hallaba en Madrid por motivos de estudios y que tenían bien justificada su ausencia de la Iglesia de Canarias. También Sempere y Guarinos corroboró este dato. Este autor escribió que Agustín Madan se dedicaba en Madrid al estudio de las lenguas griegas y hebrea. Recibía enseñanzas de fray Juan Antonio Ponce (Religioso Mínimo) quién conocía estas lenguas. Junto con Madan asistían José Rodríguez de Castro, Manuel José Marín y Juan Domingo Cativiela, ya entonces conocidos como buenos literatos. A estas sesiones de estudio también acudían otros jóvenes, reuniéndose todos los jueves y domingos para estudiar la lengua hebrea y la griega. ${ }^{8}$

Resulta interesante constatar que en la formación intelectual de Madan dejaron profunda huella los estudios que culminaron en esa permanencia en Madrid, no exclusivamente con textos académicos sino una formación que adquirió con varios eruditos que actuaban como aprendices intelectuales. Entre esas destacadas personalidades del mundo de las letras cabe mencionar a Miguel Casiri, sacerdote experto en lenguas orientales, Casimiro Flórez Canseco y Felipe Scío de San Miguel expertos en lenguas clásicas. En el aprendizaje a través de las referidas tertulias con otros eruditos literatos reflexionó sobre la renovación pedagógica. En su deseo de proyectar una enseñanza más flexible y operativa reflejaron en lecciones orales sus ansias de saber además de proyectar una exigencia metodológica. La relación con la élite intelectual y con los estudiosos de las lenguas clásicas propició el intercambio cultural, dialéctico e incluso humano.

También trabajó como Pasante de Pluma en Madrid con el abogado Vicente García Hernández, puesto donde demostró su competencia laboral. Opositó a la Canongía Doctoral de la Iglesia Catedral de Zamora y aprobó los ejercicios literarios «nemine discrepante». Miembro de la Academia de Sagrados Cánones de San Juan de Nepomuceno ejerció el cargo de Fiscal y Vicepresidente. El 14

\footnotetext{
${ }^{7}$ A.H.N. Consejos Suprimidos. Legajo 483. Expediente 7. Lista de curas retirados a sus destinos por orden de los Alcaldes de Casa y Corte (con motivo del Motín de Esquilache). Año 1766. Fol. 7 rto.

${ }^{8} \mathrm{~J}$. SEMPERE Y GUARINOS, Ensayo de una biblioteca española de los mejores escritores del reinado de Carlos III. Tomo II. Madrid, 1785-1789, 151.
} 
de marzo de 1765 se graduó de doctor en Cánones en la Universidad de Avila. En 1766 opositó a la Canongía Penitenciaria de la Catedral de Salamanca, aprobando los ejercicios «nemine discrepante». En esta misma fecha incorporó a la Universidad salamantina el grado de bachiller en Cánones y estudió Lengua griega. En 1766 recibió el nombramiento de Racionero de la Catedral de Santa Ana de Las Palmas, regresó a Canarias y tomó posesión el 6 de diciembre de 1767. ${ }^{9}$ Cuando tomó posesión de la referida ración renunció al puesto de Provisor y Vicario General Eclesiástico asignado por el arzobispo electo de Guatemala don Pedro Cortés. De esta manera, Agustín Madan tras completar estudios en tierras peninsulares volvió a Las Palmas a ocupar su prebenda de racionero en la Catedral. Sin embargo, en su mente planeaban otras aspiraciones y regresó a Madrid en 1769 con la idea de opositar a la cátedra de Hebreo de los Reales Estudios de San Isidro. Marchó sin autorización del Cabildo Catedral porque su solicitud fue desestimada y considerada una excusa para viajar a la península, ${ }^{10}$ dudando del verdadero motivo de su viaje. ${ }^{11}$ No se amilanaba ante las reiteradas presiones, seguía ilusionado con sus proyectos y su pretensión de ocupar un puesto docente en el Colegio de los Reales Estudios de San Isidro de Madrid.

En Madrid, mientras preparaba su cátedra se encontraba, en calidad de maestro interino, en la casa del Marqués de Santa Cruz de Mudela (Grande de España), José Joaquín de Silva y Bazán, instruyendo a su unigénito Francisco de Silva, Marqués del Viso. Pero se veía obligado a renunciar a su empleo de ayo, y recomendó a José de Viera y Clavijo que por aquel entonces viajaba desde Canarias a la España peninsular (arribó a Cádiz el 21 de noviembre de 1770 y llegó a Madrid el 13 de diciembre de 1770) para que le sustituyera. El propio Viera, en una carta que dirigió a Juan Antonio de Urtusáustegui, escribió: «he sucedido a Madan en la intendencia del hijo del Marqués de Santa Cruz...».12

Pese a todos los obstáculos que tuvo que sortear alcanzó su objetivo. En 1771 Agustín Madan opositó a la Cátedra de Hebreo de los Reales Estudios de San Isidro de Madrid, obtuvo la plaza de catedrático de Hebreo ${ }^{13}$ y la regentó por espacio de diez años. La formación de este isleño fue suficiente para ganar

\footnotetext{
${ }^{9}$ A.C.S.A.L.P.G.C. Libro de Señores Prebendados. Año 1767. Fol. 29 Vto.

${ }^{10}$ A.C.S.A.L.P.G.C. Libro Capitular, N. 48. Fecha 6 de junio de 1768 a 9 de abril de 1772.

${ }^{11}$ A.C.S.A.L.P.G.C. Legajo de Cartas, $n^{\circ} 5$ (1768-1784). Sin foliar. Carta fechada el día 3 de septiembre de 1771, sobre licencia de Madan.

12 A.R.S.E.A.P.T. Fondo Moure. Carta no 2 (diciembre 1770).

13 J. Simon Diaz, Historia del Colegio Imperial de Madrid. Madrid, 1952, 60-61. A la oposición para la cátedra de la lengua hebrea, celebrada el 22 de octubre de 1771, concurrieron seis opositores, a saber: Ignacio López de Ayala, Juan Domingo de Cativiela, García de Valbuena, Antonio Mangas, Manuel García y Agustín Madan.
} 
la cátedra y por ello se convirtió en el primer ${ }^{14}$ catedrático de hebreo de los Reales Estudios de San Isidro de Madrid..$^{15}$ El desarrollo de los ejercicios de la oposición fue testigo de la alta cualificación lingüística; preparación que no sufrió mermas ante las dificultades y contratiempos que tuvo que superar. Además de ser el primer docente canario en un colegio de esta envergadura y así lo reconocieron destacadas personalidades isleñas en la época. En Canarias no pasó desapercibido su nombramiento. Así lo reflejaron los hermanos Lope Antonio ${ }^{16}$ y Fernando de la Guerra y Peña ambos conocidos desde su infancia. Fernando de la Guerra y Peña en una carta fechada el 16 de enero de 1772 dirigida a su hermano Roberto escribió lo siguiente:

«...Debía ser de felicitación; por lo que me intereso tanto en que el señor don Agustín (en cuya gramática latina tuve parte y a quien estimo desde sus tiernos años) haya logrado un acomodo útil y lucido, libre de canónigos, que lo perseguían sólo porque sabía más...».17

Al mismo tiempo valoraba el mérito de la oposición y reconocía el éxito de que un canario obtuviera un puesto académico de esa categoría.

«...cuanto que nuestra tierra haya dado un rabí a la corte de España».18

Por otra parte, a pesar de las ya referidas dificultades con el cabildo catedral, tuvo influencia y mantuvo relaciones en círculos cortesanos, sobre todo en las cuestiones relativas a la enseñanza de las lenguas clásicas. Su relación con el mundo cultural madrileño fue muy intensa a través de tertulias y estudios; se relacionaba con lo más granado de los lingüistas españoles, expertos en lenguas clásicas, y en sus reuniones ahondaba en el estudio de la lengua hebrea. Divulgador de los nuevos planteamientos de las lenguas clásicas, deseoso de que progresaran sus estudios, puso todo su empeño en la docencia del hebreo.

En 1772 fue nombrado Beneficiado de la parroquia de Santa Catalina de Sevilla. Más tarde le nombraron Censor del concurso oposición de la Cátedra de

\footnotetext{
${ }^{14}$ A.G.S. Sección de Gracia y Justicia. Inventario 64.Legajo 972. Resolución de El Consejo, 22 de octubre de 1771. «El Consejo. 22 de octubre de 1771. En vista de lâ adjunta censura de los examinadores nombrados para la oposición â la Maestria de Lengua Hebrea, que se restablece en el nuevo Real Sn. Ysidro de Madrid, y de la Graduacion, que, del respectivo merito, y circunstancias de los opositores, han hecho los Ministros Comisionados para asistir â los examenes; propone el Consejo para dicha Maestria. En 1. Lugar â dn. Agustín Madam. en 2. â dn. Antonio Mangas.en 3. â dn. Manuel García. El Rey nombra al del primer lugar».

15 J. SimOn DiAZ, Historia del Colegio Imperial, opus cit., 61-66.

${ }^{16}$ L.A. Guerra Y PeÑa, Memorias: Tenerife en la segunda mitad del siglo XVIII. Tomo II. Las Palmas, 2002,19.

${ }^{17}$ A.R.S.E.A.P.T. Fondo Moure. Legajo de Cartas.

18 Ibidem.
} 
la Lengua Griega, vacante en los Reales Estudios en 1777. También en 1778 le designaron Censor y Examinador de la Pasantía de la misma Lengua. No obstante, no rompió relaciones con el Cabildo Catedralicio de Canarias y en 1779 fue designado su representante en la Corte. Realizaba gestiones para la Iglesia de Canarias en Madrid ocupándose de la gestión de diversos asuntos. Mantenía correspondencia fluida y le asignaban multitud de encargos. Asimismo en 1780 obtuvo una Canongía en la iglesia de Canarias. Además fue miembro y apoderado de la Real Sociedad Económica de Amigos del País de Las Palmas. Igualmente fue socio y diputado de la Real Sociedad de Amigos del País de Tenerife. Se encargaba en Madrid de gestionar asuntos y encargos diversos de ambas entidades patrióticas.

Testimonio de su dedicación docente fueron las obras de carácter didáctico que escribió. En 1773 publicó el trabajo «Pro prima disciplinae tentamine, publico doctorum judicio proponuntur theoremata de necessitate, et utilitate linguae hebraicae, de origine scripturae in genere, de Elementis, Idiotismis ejusden linguae».19 Más tarde en 1776 editó «Exercitationes philologicae de institionibus linguae Santae, ac hebraici textus interpretatione, quas in regio archigynm, matrit». ${ }^{20}$ En 1781 redactó el «Discurso acerca de la negligencia del estudio de las lenguas Sagradas en España, donde se proponen los medios para fomentarlo». También localizamos dos manuscritos autógrafos referidos a monedas antiguas. Estos dos manuscritos no son obras originales sino anotaciones sacadas de otros libros. ${ }^{21}$

\section{El PODER DEL CONOCIMIENTO: LA CONSULTA DE CAMPOMANES}

En España, igual que sucedió en otros estados europeos, los ilustrados confiaban en la educación y en los beneficios que reportaba para alcanzar la reforma social. ${ }^{22}$ Sin embargo, esa actitud de optimismo pedagógico contrastaba con la caótica situación educativa, la escasa cualificación de los maestros y la ignorancia metodológica ${ }^{23}$ generalizada en todos los niveles. Los gobernantes estimaban que la educación era competencia del poder público. El gobierno debía difundir la cultura y resolver los problemas de ignorancia generalizando la difusión de los conocimientos útiles. Campomanes proclamó los conocimientos úti-

\footnotetext{
${ }^{19}$ F.A.B.U.L.L. Papeles Varios. Tomo 47

20 Ibidem.

${ }^{21}$ F.A.B.U.L.L. 13 de febrero de 1782. Seis folios escritos sin numerar. A.M.C. Colección Roja. Tomo I. Sin foliar.

${ }^{22}$ M. Domínguez LÁzAro, La educación española en la segunda mitad del siglo XVIII. Revista Española de Pedagogía, Vol. 43, n ${ }^{\circ}$ 67, 71-90.

${ }^{23}$ E. Martínez RuIz y Otros, La España Moderna. Madrid, Itsmo, 1995, 484.
} 
les refiriendo sus ventajas en «las naciones sabias e industriosas». Se inclinaba por difundirlos para alcanzar el progreso económico, entendiendo indudablemente la interrelación entre educación y desarrollo, tesis de plena actualidad, pues ayer como hoy siguen generando progreso.

Pedro Rodríguez de Campomanes y Pérez (1723-1802), abogado, político, economista, historiador, tuvo una intensa actividad. Ocupó diversos puestos en la administración del estado, impulsó las Reales Sociedades Económicas y se interesó por la educación, aunque ha sido una de las facetas menos conocida en la historiografía. Igualmente escribió varios libros, informes, memoriales, discursos... Uno de los más célebres, por su proyección social, fueron el «Discurso sobre el fomento de la industria popular» (1774) así como «Discurso sobre la educación popular de los artesanos» (1775) y sus Apéndices (1775-1777). La carrera política de este ministro y gobernador del Supremo Consejo de Castilla, se sitúa entre 1762 y 1791, año en que comienza su declinar con la prisión de Cabarrús, y su relevo en 1791, aunque continuó de Consejero de Estado hasta 1802.

En el hilo de los ilustrado tomó cuerpo la crítica a las enseñanzas tradicionales, a la filosofía y a la especulación abstracta, que al decir de Campomanes «toda la atención se ha llevado al estudio de las especulaciones abstractas, y aún en éstas ha habido la desgracia de que en las materias de ningún uso y vanas haya sólido ponerse más ahínco que en los conocimientos sólidos y usuales». ${ }^{24}$ En el pensamiento de los ilustrados está presente, de forma constante, la preocupación por renovar contenidos, y así aparece en el Discurso sobre la Educación Popular de Campomanes, en la Memoria sobre la Educación Pública de Jovellanos y en la Instrucción Reservada de Floridablanca. Resultaba urgente la reforma de la enseñanza en todos sus niveles, mejorar la formación de maestros y la calidad educativa, al tiempo que renovar la metodología, toda vez que la práctica se regía por la rutina. A la rancia ignorancia didáctica, se añadía la ausencia de experimentación y observación y unos contenidos impregnados por la escolástica y el aristotelismo. De esta manera la reforma alcanzó a los estudios superiores diseñando nuevos planes de estudio..$^{25}$

Ante la decadencia intelectual y educativa se abordó la reforma de la enseñanza ${ }^{26}$ con la secularización de oficio de maestro de primeras letras y exten-

\footnotetext{
${ }^{24}$ P. RodrígueZ De CAmpomanes, Discurso sobre la educación popular de los artesanos y su fomento» Madrid, 1775, p. 35. Al respecto resulta bastante elocuente su afirmación «más útil en el orden civil al género humano la invención de las agujas de coser, instrumento de tanto uso, que debe preferirse a la lógica de Aristóteles y a un gran número de sus comentadores, los cuales han sido en España más comunes que las fábricas de agujas».

25 A.Florintán, Historia de España en la Edad Moderna. Barcelona, Ariel, 2004, 733.

${ }^{26}$ L. FERnÁNDEZ GiL y otros, La cultura española en la Edad Moderna. Madrid, Itsmo, 2004, 484.
} 
sión a los niños de las capas populares. En la enseñanza secundaria, monopolizada por el latín, se introdujo el castellano y mejoraron los contenidos. La enseñanza universitaria era similar, obsoleta, bloqueada la ciencia moderna y se pretendía desplazar el control de la iglesia. Campomanes ponía énfasis en la instrucción, en «aquellos conocimientos que son necesarios para ser útiles al Estado». La política educativa no cubrió las aspiraciones ilustradas. La ciencia moderna se observaba como «un enemigo potencial» porque indudablemente el control del clero era efectivo. Tanto Pablo de Olavide como Gregorio Mayáns coincidían con la necesidad de reforma universitaria y que ningún religioso enseñara en la universidad. ${ }^{27}$ Sin embargo, la reforma universitaria fue moderada y sólo introdujo algunas novedades sobre la base escolástica-aristotélica, pequeñas incursiones científicas en suma. El afán pedagógico ${ }^{28}$ estuvo presente en casi todos los ilustrados, incluidos los religiosos, entre ellos, Feijóo, que escribió sobre la desfasada praxis. Criticaron los métodos pedagógicos, a pesar del revuelo causado entre los retardatarios que rechazaban las innovaciones.

Observamos la compleja y polifacética personalidad de Campomanes, no sólo preocupado por los asuntos de estado, también por la cultura y la educación, así como su proyección en mejorar la instrucción, por combatir la ignorancia. En su múltiple actividad, también estudioso de las lenguas clásicas, una de las ocupaciones menos difundida, preocupado por los estudios lingüísticos en los centros religiosos y civiles, convencido de los beneficios que reportaban, efectuó repetidas consultas a distintos expertos. Ya en su solicitud de ingreso en la Real Academia de la Historia (1748) afirmaba «estar sobradamente instruido» en los idiomas francés e italiano tener «alguna tintura del griego y del hebreo». Aprendió las lenguas clásicas en Madrid, entre los años 1748-1751, con los maestros de renombre de aquel entonces. Fue discípulo de Miguel Casiri (presbítero siromaronita) que le enseñó árabe y de José Carbonell que lo instruyó en griego y con probabilidad en hebreo. Más tarde fue nombrado Director de la Real Academia de la Historia.

Pedro de Campomanes leía los clásicos y al contrario que Feijóo no creía que fuera una pérdida de tiempo. Su admiración y afición por los estudios históricos y los autores clásicos le condujo a dedicarse a su estudio. Estaba convencido de los beneficios de la lectura de los clásicos y se propuso luchar contra la ignorancia y recuperar el buen gusto. Reconocía como imprescindible el conocimiento de la lengua griega y árabe, de tal manera que algunos autores han identificado a este ministro ilustrado como la persona que más se interesó

\footnotetext{
27 E. Martínez Ruiz y Otros, La España Moderna. Opus Cit. 485.

${ }^{28}$ P. Molas Ribalta, Edad Moderna (1474-1808). Madrid, Espasa-Calpe, 1988, 536.
} 
por los estudios helenísticos en España. ${ }^{29}$ Incluso intentó reformar los planes de estudios universitarios introduciendo estas lenguas. El interés por el griego así como su introducción gradual en la enseñanza superior le conminó al fracaso. Pretendió reformar los planes de estudios universitarios introduciendo las mencionadas lenguas, pero la tarea emprendida no se reveló como exitosa. Únicamente en los Reales Estudios de San Isidro de Madrid, donde se implantó en 1771, tuvo un buen nivel de aceptación. En los centros religiosos y seglares introdujo también la enseñanza del griego, pero su fracaso fue evidente en ambos casos. Los resultados tan nefastos no contentaron a Campomanes pero tampoco lo desanimaron y cuestionó la metodología didáctica de esta lengua. De este modo consultó a los profesores de los Reales Estudios de San Isidro y dos de los profesores que integraban el claustro se encargaron de analizar las causas que impedían la evolución académica de estas materias. El catedrático de griego Casimiro Flórez Canseco elaboró el «Método para enseñar y aprender la lengua griega». El catedrático de hebreo Agustín Madan examinó los motivos del retraso de la lengua hebrea en el «Discurso acerca de la negligencia del estudio de las lenguas sagradas en España». ${ }^{30}$ En el manuscrito propuso los medios para fomentar el aprendizaje lingüístico y constituye un buen exponente didáctico de la lengua hebrea de la época. ${ }^{31}$ Constituye el texto que mejor condensaba su planteamiento educativo con respecto a las lenguas clásicas. En él profundizó en las directrices metodológicas y didácticas de la lengua hebrea, al tiempo que proporcionó los recursos aplicables a su enseñanza. En su argumentación demostró sus conocimientos y su cercanía a las tesis jansenistas, afín con el pensamiento de otros docentes de los Reales Estudios. Esta consulta que efectuó Campomanes a Agustín Madan nos revela la estima que había alcanzado este ilustre profesor canario en la Corte. Cualificado en el conocimiento de las lenguas clásicas y en el arte de enseñar, incitaron a un ministro de Carlos III a solicitar su asesoramiento, con la finalidad de promocionar estos estudios.

\section{LA VOCACIÓN DOCENTE: SU PROPUESTA DIDÁCTICA}

No ignoramos que Agustín Madan fue un preceptista que estudió y promovió el saber y trató de establecer las reglas de aprendizaje de una lengua que

${ }^{29}$ L. Gil Fernandez, Campomanes un helenista en el poder. Seminario Diego Hurtado de Mendoza. Madrid, 1976.

MEMORIAL de ingreso que escribe D. Pedro Rodríguez Campomanes, Abogado de los Reales Consejos y del Ilustre Colegio de esta Corte, con el motivo de su admisión en la Real Academia Española de la Historia, a quien la dedica, consagra y ofrece, leída el 5 de abril de 1748. Boletín de la Real Academia de la Historia, Madrid, LXXVIII, 1921, 460-461.

${ }^{30}$ A.F.U.E. Fondo Campomanes. Documento n 31. Legajo 17/6. Año 1781.

${ }^{31}$ A.F.U.E. Fondo Campomanes. Documento no 20. Año 1781. Sin foliar. 
consideraba crucial. El «Discurso acerca de la negligencia del estudio de las lenguas Sagradas en España, donde se proponen los medios para fomentarlo», no era un estudio muy amplio pero expresó en él su pensamiento y exigencia metodológica. Madan había sido aprendiz de intelectual, agregado a las tertulias madrileñas cuando residía en la calle El Prado. La complejidad de las influencias era auténtica. En ella se vislumbraba el porvenir del hebreo, erudición que dirigía a un grupo de cierto nivel cultural y social. En su multiplicidad, también luchaba contra la ignorancia y polemizaba por el descuido de estos estudios. Sus comentarios demostraban tanta cultura como inteligencia convencido de la necesaria reforma de estudios, la incorporación de la lengua y del papel que desempeñaba el hebreo en España. Como docente y pensador aplicó la pedagogía a su campo del saber, es decir, a las lenguas.

Con el objetivo de promocionar esta lengua Agustín Madan programó y planificó la materia, distribuyendo sus contenidos y actividades. Con agudeza observó la decadencia del hebreo en España y con ella de los estudios bíblicos. Consideraba que había que elevar el prestigio de esta lengua y garantizar la formación de estudiosos para difundir y promocionar su conocimiento, como quiera que su conocimiento era fundamental para conocer la «palabra revelada». Por este motivo se interesó por el estudio de la Sagrada Escritura Pretendía fomentar el aprendizaje de la lengua hebrea como actividad básica para la comprensión del «texto sagrado», porque desde su punto de vista para interpretar la Biblia había que dominar necesariamente la lengua hebrea. ${ }^{32}$ En su pensamiento docente el hebreo se hallaba estrechamente unido a los dogmas de la religión cristiana. Por este motivo indicaba que la forma óptima de traducción era recurriendo al texto primitivo de la Biblia. Asimismo, se preocupaba por el descrédito que sufrían algunas versiones hebreas. Además su situación de eclesiástico le confiere unos criterios concretos para valorar la importancia, superando los niveles de utilidad que ésta pudiera tener. En todo su discurso reiteraba que, para conocer la Sagrada Escritura, se precisaba el estudio del hebreo, justificado además porque estaban escritos en esta lengua casi todos los libros del Viejo Testamento. 33

En este orden argumentaba que la Teología era un pilar básico para sustentar los estudios bíblicos y el conocimiento histórico de la iglesia primitiva. En su proyecto educativo, la Teología y la formación de los teólogos ocupaba un lugar destacado. Una manera de superar las posibles deficiencias de los teólogos consistía en formar grandes maestros. ${ }^{34}$ En este sentido coincidía con el pensamiento de Gregorio Mayáns «las lenguas orientales totalmente se ignoran; i

\footnotetext{
${ }^{32}$ Ibidem. Documento ${ }^{\circ} 20$. Sin foliar.

${ }^{33}$ Ibidem.

${ }^{34}$ Ibidem.
} 
hasta que no se renueven no habrá theólogos grandes» destacando la necesidad de que estudiaran como mínimo «medio año de hebreo y medio año de griego». Incluso, sentía cierta aversión por los escolásticos que desacreditaban el estudio del hebreo y del latín. De este modo, Mayáns manifestaba:

«Escrivir contra las lenguas hebrea i griega, es escrivir contra las lenguas originales con que está escrita la divina palabra, cosa que no haría si no fuera ignorante de primera clase». ${ }^{35}$

Mayáns pensaba que la decadencia de los estudios de hebreo en España estaba causada por la prohibición bibliográfica. ${ }^{36}$ Estimaba que al censurar la bibliografía hebrea se contribuyó a su decadencia, por ello insistía en la necesidad del conocimiento del hebreo para estudiar Teología. Entendía que todas las personas debían conocer la Sagrada Escritura, no limitarla a los eclesiásticos e intelectuales ${ }^{37}$ Asimismo reconocía a la Biblia como texto único y proponía como objetivo el conocimiento literal de la misma, pues la grandeza radicaba en seguir la doctrina cristiana de acuerdo con la Sagrada Escritura. De esta forma Mayáns justificó la importancia de la lengua hebrea:

«Estando escritos originalmente en Hebreo casi todos los libros del

Viejo Testamento, es de suma importancia aprender la lengua hebrea...

pues el fin de aprender esta lengua es entender la Sagrada Escritura...».38

Por su parte Agustín Madan había demostrado su competencia y dominio del método para la enseñanza de dicha lengua. ${ }^{39}$ Cualificado en el conocimiento de las lenguas clásicas y en el arte de enseñar incitaron a un ministro de Carlos III a solicitar su asesoramiento con la finalidad de promocionar dichos estudios. Madan inició su estudio efectuando un balance sobre el estado de la lengua hebrea. Analizó su evolución en los siglos anteriores, iniciando su recorrido en el siglo XVI. Manifestaba que en el Siglo de Oro el Cardenal Jiménez compuso la Biblia Políglota y de tal manera el prestigio de esta obra repercutió en la sociedad española que se catalogó entre las naciones cultas. Así mismo, alegaba que Felipe II y sus ministros se interesaron por esta lengua:

«como tributo debido â lo sublime de nuestra religion, que se contiene en la sagrada Biblia, y mui propio de una Nacion en qe. se conserva con tanta pureza el deposito dela fee Christna».

\footnotetext{
${ }^{35}$ Escrito por Mayáns en una carta dirigida a Felipe Seguer, fechada en Oliva el 16-VI-1759. Documento citado por A. Mestre SANCHIS, Ilustración y Reforma de la Iglesia: Pensamiento político-religioso de don Gregorio Mayáns y Siscar (1699-1788). Valencia, Ayuntamiento de La Oliva, 1968.

36 Ibidem, 329.

37 Ibidem, 330-331.

38 Ibidem, 329.

${ }^{39}$ A.H.N. Sección Consejos. Legajo 5441. «Methodo para la Lengua Hebrea». Dn. Agustín Madan Cathedco. de Hebreo.
} 
No dudaba que para redactar esta obra en el idioma hebreo se invirtió mucho dinero; la cuantía económica a la que ascendió la biblia era importante, sin embargo, no entendía cómo, después de abonar ese elevado coste, no se utilizaban estos ejemplares ni mucho menos comprendía esta actitud en el siglo ilustrado. De ahí la decadencia y desprestigio al que había llegado la enseñanza del hebreo. Por ello planteó diversas cuestiones, entre ellas:

\footnotetext{
- «iMiraremos pues con indiferencia que se aprovechen de nuestros trabajos las Naciones estranjeras, y aún los enemigos de nuestra religión?»

- «iSeremos, como los Judíos, que guardan como Depositarios los libros de la Escritura sin entenderla?»
}

Expresaba que el monarca Carlos III restableció los estudios de las lenguas griega, árabe y hebrea «y de la version del texto original de la Biblia» en los Reales Estudios para que tomaran ejemplo otras instituciones educativas. Sin embargo, observaba que en los diez años que llevaba desempeñando la cátedra de hebreo no progresaba el estudio de esta lengua. Consideraba que una forma de promocionarla era incluirla en los estudios de Teología por su utilidad para la interpretación de la Sagrada Escritura. Igualmente se implicó en buscar posibles soluciones para revalorizar el estudio del hebreo e incentivar al alumnado. Su punto de referencia era Francia, país al que se remitía continuamente. Además se preocupaba por el descrédito de algunas versiones de la Biblia debido a las alteraciones del texto. Indicaba así mismo que la forma óptima de traducción era recurriendo al texto primitivo de la Biblia. En su pensamiento docente el hebreo se hallaba estrechamente unido a los dogmas de la religión cristiana. En su empeño por promover esta «lengua santa» planificó la materia, sus contenidos, actividades... Además su situación de eclesiástico le confirió unos criterios concretos para analizar la importancia de esta lengua superando los niveles de utilidad que ésta podía tener. Como eclesiástico revalorizó esta lengua, entendiendo que no se consolidó en la sociedad de la época. De manera que denunció la decadencia de la lengua sagrada en España y con ella de los estudios bíblicos. Sorprende observar la importancia que atribuyó al estudio de la Sagrada Escritura. Había notado la declinación de los estudios bíblicos en España y por ello pretendía fomentar el aprendizaje de la lengua hebrea como actividad básica para la comprensión del texto sagrado porque para interpretar la Biblia había que dominar la lengua hebrea. El principal fin de su estudio era conocer el sentido literal de la palabra revelada y el conocimiento perfecto de la Sagrada Escritura exigía el estudio de la lengua hebrea. Desde su posición docente luchó por fomentar el estudio de la lengua erudita para mejorar el conocimiento de la palabra revelada del Antiguo Testamento. El estudio de esta lengua quedaba justificado exclusivamente por el conocimiento de la Sagrada Escritura, pues originalmente estaban escritos casi todos los libros del Viejo Testamento en hebreo. De ahí la importancia de las lenguas eruditas o lenguas orientales. 
Agustín Madan propuso algunas soluciones didácticas para promocionar los estudios de la lengua hebrea. Recomendaba un texto escrito en castellano para el aprendizaje de la lengua sagrada. Su método de enseñanza se basaba en el estudio de la sintaxis simple y la sintaxis figurada, además de la traducción de los profetas. Como material de trabajo necesitaban tres cuadernos: 1- Para realizar las anotaciones sobre la gramática. 2- Para el vocabulario no recomendaba emplear el diccionario, sino apuntar las palabras y los diferentes significados que le han asignado los autores. Estas voces las memorizarían progresivamente los alumnos; calculaba que aprenderían diez voces diarias lo que representaba un cómputo total de siete meses. 3- Para incluir las observaciones pertinentes sugeridas por el catedrático. El alumnado, según estimaba, en tres meses podía iniciar la traducción del Libro del Génesis, y paulatinamente los Libros de los Profetas y los Poéticos. Su principio pedagógico se reforzaba en la enseñanza y difusión de esta lengua. Se preocupa por la asimilación de los contenidos, por memorizar y conocer la lengua sagrada. Sin duda, un estudio que llevaba implícito un aprendizaje religioso.

En las universidades españolas de aquel entonces se impartían diferentes cursos pero no había enseñanzas específicas de Hebreo y versión de la Sagrada Escritura. Agustín Madan, desde su posición de catedrático de Hebreo de los Reales Estudios de San Isidro de Madrid, se preocupó por la inclusión de su materia en los currícula universitarios. Consideraba que esta lengua quedaba relegada a un orden secundario y éste no era el objetivo propuesto por el rey con respecto a esta enseñanza, cuando fue restituida por Real Decreto en 1770. Estimaba que el verdadero problema se planteaba por la mentalidad imperante respecto a la difícil utilidad de esta lengua. Esta opinión generaba un escaso número de alumnos dedicados a su estudio y suponía una involución en su enseñanza. Así, la motivación del alumnado y el reconocimiento de la importancia de esta lengua garantizaría su éxito. Desde su óptica, el texto hebreo era interesante y constaba de varios libros: «Pentateucho, los historiales, los de los Psalmos, canticos y Proféticos». Proponía como posible solución que el monarca incentivara esta enseñanza, estimulando a los alumnos para que estudiaran la lengua hebrea. Como docente se interesaba por la enseñanza y aprendizaje de la lengua hebrea, de la religión y la teología. ${ }^{40}$

Los métodos educativos y el contenido de la propia lengua hebrea sintonizaban con la filosofía de la razón que envolvía las ideas del dieciocho. Sin embargo, se imponía «la diosa de la razón» y los nuevos pensadores perseguían nuevas formas de enseñanza guiados por modelos más coherentes y conectados

\footnotetext{
${ }^{40}$ A.R.S.E.A.P.T. Fondo Moure. Legajo de Cartas. Sgn. 20/9. Promoción del hebreo. Madrid 30 de octubre de 1777.
}

Hispania Sacra, LXIII

127, enero-junio 2011, 185-204, ISSN: 0018-215-X 
con la naturaleza humana. ${ }^{41}$ Madan, como excelente profesional, respondió desde la vertiente progresista por su área de conocimiento frente al creciente desinterés del alumnado que no encontraba sentido ni utilidad a su aprendizaje más allá del estrictamente religioso. Indudablemente, la lengua hebrea no tenía otra misión que la interpretación de los textos sagrados como el mismo profesor anuncia. Para un país que iniciaba el proceso de secularización y la sociedad buscaba otros horizontes educativos, se justificaba el escaso número de alumnos que acudían al aula de hebreo. Después de enumerar los principales argumentos que el primer catedrático de hebreo de los Reales Estudios presentó a modo de solución a Campomanes, es preciso tener presente su condición de eclesiástico para comprender su visión dogmática de la materia. Con fecha de 18 de febrero de 1781 Agustín Madan remitió al Conde de Campomanes su «Discurso acerca de la negligencia del estudio de las lenguas sagradas en España, donde se proponen los medios para fomentarlo».42

«Por el superior influxo de un Magistrado de primer orden amante de la patria, y de las letras, me hallo impelido â escrivir sobre los medios, y arbitrios para promover en los Rs. Estudios de Sn. Ysidro, y demas del Reyno el estudio de las lenguas sagradas, especialmente dela hebrea cuya catedra he regentado pr. 10 años».

Sin embargo las aportaciones didácticas de estos trabajos no tuvieron efectividad en la práctica, no entraron en vigor y, por tanto, no cumplieron su objetivo de renovación pedagógica. ${ }^{43}$ Además de lo anterior, decir que las reformas de los ilustrados no alcanzaron los objetivos propuestos. Fueron poco originales aunque escribieron mucho, motivo por el cual buena parte de sus planteamientos no se aplicaron en la práctica y sus proyectos no superaron el plano documental y de buenos propósitos. La Ilustración fue un fenómeno complejo

\footnotetext{
${ }^{41}$ A. Capitan Diaz, Historia del pensamiento pedagógico europeo. Dykinson. Madrid, 1984, 585 y ss.

N. Abbagnano y A.Visalberghi, Historia de la Pedagogía. Fondo de Cultura Económica. Madrid, 1995, 369-386.

${ }^{42}$ A.F.U.E. Fondo Campomanes. Legajo 17/6. Carta que envia Agustín Madan al Conde de Campomanes acompañando su «discurso».

«Yltmo. Sor. Mui Sor. mio, Paso à las manos de V.Y. el papel que he formado, mediante la insinuación de V.Y., para manifestar los medios depromover el estudio del Hebreo en nuestras escuelas; me he extendido enla introduccion mas de lo regular porque me parece ser el principio mas seguro para excitar dho estudio eldemostrar su utilidad; Si estuviese del agrado de V.Y., lo haré copiar por un escribiente habil que evite los defectos deortografía que se hallan en este. Ynterim quedo mui ala obediencia de V.Y. deseando sus ordenes y que nrô Señor guarde su importante vida ms. as.

Madrid y Febo.18 de 1781

Yltmo. Sor.

Blmo. de VYltma. su mas sego. servor. y Capn.

Agustín Madan

Yltmo. Sor. Conde de Campomanes»

${ }^{43}$ L. Gil Fernandez, Campomanes. Opus Cit. 71.
} 
lleno de buenas intenciones, pues el setecientos se caracterizó por una política reformista si bien había que matizar que se caracterizó por «más proyectos que realidades». De manera que no tenemos constancia de que este informe se aplicara, y no dudamos que, como tantos otros, no superara el plano escrito.

Un mérito lo constituye el convencimiento y la insistencia con que repetía que el hebreo era indispensable para conocer la palabra de Dios, fuente de vida espiritual y expresión de belleza literaria. Además, consideraba que conocer la palabra de Dios no se reducía a los predicadores que de forma exclusiva interpretaban la Biblia, sino que era el deber de todo cristiano, pues en ella debía buscar la norma de conducta moral ya que era el principio de su filosofía moral. El pensamiento de Madan sobre la disciplina que enseñaba resultaba un tanto dispar con las ideas educativas que se respiraban en la época en el contexto europeo. Si bien se remitió a Francia para exaltar la preponderancia de la lengua hebrea tal como allí se enseñaba, no hizo mención al movimiento cultural que allí se desarrollaba ni al nuevo concepto de la sociedad y de la vida. Este movimiento, arropado bajo la denominación de Ilustración, pregonaba que si el hombre recibía una educación apropiada alcanzaría la felicidad. Así, desterrando la ignorancia la humanidad caminaría por los senderos de la felicidad. La filosofía de la Ilustración proyectaba la cultura para el pueblo en un intento de disipar la superstición difundiendo conocimientos útiles al margen de la educación de la élite.

En este sentido Madan no construyó principios pedagógicos nuevos, sin embargo, innovó con sus ideas. Se preocupó por la asimilación de los contenidos, memorizar y conocer la lengua sagrada, lo que llevaba implícito un mensaje religioso. Si bien su método de enseñanza encajaba perfectamente con los métodos empleados por las órdenes religiosas, pues, enseñaba básicamente retórica. Su mentalidad como eclesiástico coincidía más con las prácticas educativas tradicionales y de las instituciones religiosas. Los métodos educativos y el contenido de la propia lengua hebrea chocaban con la filosofía de la razón que envolvía las ideas de los hombres del dieciocho. Se imponía «la diosa de la razón» y los nuevos pensadores perseguían nuevas formas de enseñanza guiados por modelos más coherentes y conectados con la naturaleza humana. En esta dirección señalaba Rousseau, el padre de la pedagogía, la enseñanza conveniente para la humanidad. Este tipo de enseñanza lo dejó entrever en su obra «El Emilio o de la Educación» publicada en 1762, donde enuncia las tesis principales sobre la educación. ${ }^{44}$ Para su discípulo Emilio proponía un aprendizaje flexible basado en la naturaleza humana y la libertad, como réplica a la educación de la época. ${ }^{45} \mathrm{~A}$ pesar de este desfase con el progresismo educativo de la

44 J. J. Rousseau, El Emilio o de la Educación. Barcelona, Bruguera, 1979.

45 M. T. VAzquez Prada, La Historia de la Educación. Barcelona, PPU, 1989, pp. 210-266. 
época, Madan se imponía como un excelente profesional, respondiendo por su área de conocimiento frente al creciente desinterés del alumnado que no encontraba otro sentido ni utilidad a su aprendizaje más que el estrictamente religioso. Indudablemente, la lengua hebrea no tenía otra misión que la interpretación de los textos sagrados como el mismo profesor anunció. Para un país donde la secularización se encuentra en vías de desarrollo y la sociedad buscaba otros horizontes educativos, estaba justificado el escaso número de alumnos que acuden al aula de hebreo.

\section{CONCLUSIONES}

Pocos datos recoge la historiografía relativos a Agustín Madan. El historiador José de Viera y Clavijo citó a Madan en la Historia de Canarias, incluyó sus datos en la Biblioteca Canaria y en alguno de sus diversos trabajos. No fue exhaustivo en su tratamiento y no lo mencionó cuando refería a los isleños residentes en Madrid. Sin embargo, tenían afinidades intelectuales y su amistad no se rompió en el curso de los años. Se relacionaban desde la juventud cuando coincidieron en La Laguna y posteriormente se reencontraron en Madrid y en Las Palmas. Las cartas personales evidencian el aprecio que se profesaban, aunque ambos tenían personalidades muy distintas. Viera más extrovertido y viajero gozó de reconocimiento, mientras Madan pasó desapercibido porque era más retraído y poco proclive a las relaciones externas.

La recomendación de Madan para que Viera y Clavijo lo sustituyera al frente de la educación del hijo del Marqués de Santa Cruz de Mudela, José Joaquín de Silva y Bazán, influyó en la trayectoria del ilustre historiador José de Viera y Clavijo. Aunque la acción de Madan haya pasado inadvertida, fue un hecho que sin duda ejerció un visible influjo sobre la vida de éste polígrafo. Se instaló como ayo en casa de esta familia noble para ejercer la docencia, coyuntura favorable que le facilitó viajar por Europa. Dichos viajes de José de Viera por Europa acompañando a su alumno Francisco de Silva, Marqués del Viso, fueron una oportunidad para conocer eminentes personalidades, al tiempo que le permitieron completar su vasta formación. Madan prefería el estudio a la vida en el palacio, algo que a Viera le fascinaba.

Fueron muchos los obstáculos que tuvo que sortear Agustín Madan a lo largo de su vida como miembro del clero. Pero no se arredró y puso todo su empeño en lograr sus propósitos, incluso ignorando las prohibiciones. La atracción de Madrid para el joven inquieto y estudioso despertó los recelos de la jerarquía eclesiástica que censuró su actividad e intentó frenar sus objetivos. No sólo fue perseguido por leer libros prohibidos sino que le negó permiso para desplazarse a Madrid para opositar al Colegio de los Reales Estudios. Aunque gestionaba 
los asuntos del Cabildo Catedral durante su estancia en Madrid no recibió el reconocimiento que merecía. Esa conducta del clero capitular contrastaba con la estima que poseía como profesor en el Colegio de los Reales Estudios de San Isidro de Madrid.

El ministro de Carlos III Pedro Rodríguez de Campomanes, consecuente con los postulados ilustrados, trató por todos los medios de impulsar la educación y corregir sus defectos. Éste fue uno de los motivos que le movió a solicitar a Agustín Madan que realizara un estudio sobre la forma de promocionar las lenguas clásicas. En su estudio plasmó su pensamiento didáctico planteando la renovación del sistema de enseñanza-aprendizaje en el «Discurso acerca de la negligencia del estudio de las lenguas sagradas en España». Probablemente las aportaciones didácticas quedaron a nivel teórico y no cumplieron su objetivo de renovación pedagógica, como otros tantos propósitos de los ilustrados. Sin embargo, esta solicitud prueba el reconocimiento de su categoría profesional, de la sólida formación que poseía con amplios saberes filológicos además de su preparación teológica y filosófica. Sin duda, el siglo pedagógico fue también el suyo.

Hilvanar estar líneas no ha resultado tarea fácil, dada la dispersión de las fondos documentales, pero su estela ha permitido indagar en distintas fuentes de donde se han extraído datos valiosos. Sirvan estás páginas de reconocimiento a una figura progresista del clero isleño -estudioso y trabajador infatigableincomprendida en los escenarios vitales que transitó.

\section{FUENTES DOCUMENTALES:}

Abreviaturas correspondientes a los nombres de los Archivos:

AFUE-Archivo de la Fundación Universitaria Española. Fondo Campomanes.

AGS-Archivo General de Simancas

AHN- Archivo Histórico Nacional

ACSALPGC-Archivo de la Catedral de Santa Ana de Las Palmas

AHDLPGC-Archivo Histórico Diocesano de Las Palmas de Gran Canaria

APNSPF- Archivo Parroquial de Nuestra Señora de la Peña de Francia

ARSEAPT-Archivo de la Real Sociedad Económica de Amigos del País de Tenerife

FABULL- Fondo Antiguo de la Biblioteca de la Universidad de La Laguna

EMC- El Museo Canario 